\title{
Topological Floquet Phases in Driven Coupled Rashba Nanowires
}

\author{
Jelena Klinovaja, ${ }^{1}$ Peter Stano, ${ }^{2,3}$ and Daniel Loss ${ }^{1,2}$ \\ ${ }^{1}$ Department of Physics, University of Basel, Klingelbergstrasse 82, CH-4056 Basel, Switzerland \\ ${ }^{2}$ RIKEN Center for Emergent Matter Science, Wako, Saitama 351-0198, Japan \\ ${ }^{3}$ Institute of Physics, Slovak Academy of Sciences, 84511 Bratislava, Slovakia
}

(Received 13 October 2015; published 28 April 2016)

\begin{abstract}
We consider periodically driven arrays of weakly coupled wires with conduction and valence bands of Rashba type and study the resulting Floquet states. This nonequilibrium system can be tuned into nontrivial phases such as topological insulators, Weyl semimetals, and dispersionless zero-energy edge mode regimes. In the presence of strong electron-electron interactions, we generalize these regimes to the fractional case, where elementary excitations have fractional charges $e / m$ with $m$ being an odd integer.
\end{abstract}

DOI: 10.1103/PhysRevLett.116.176401

Introduction.-Topological effects in condensed matter systems have attracted attention for many years. From the quantum Hall effect over topological insulators (TIs) [1-12] and Weyl semimetals [13-17], to Majorana fermions [18-38] and parafermions [39-49], the interest is driven both by fundamental physics and the promise for topological quantum computation. Despite the many proposals for topological systems, the search for the most optimal material still continues unabated.

While most studies were focused on static structures, it has recently been proposed to extend topological phases to nonequilibrium systems, described by Floquet states [50-70]. Remarkably, this approach no longer relies on given material properties, such as strong spin orbit interactions (SOIs), typically necessary for reaching topological regimes, but instead allows one to turn initially nontopological materials such as graphene [50] and non-bandinverted semiconducting wells into TIs [52] by applying an external driving field.

An even bigger challenge is to describe topological effects that involve fractional excitations. This requires the presence of strong electron-electron interactions. However, given the difficulties in the search for conventional TIs, it would be even more surprising to expect such phases to occur naturally. Moreover, even if they existed, twodimensional (2D) systems with electron-electron interactions are difficult to describe analytically and often progress can come only from numerics [61].

Here, we circumvent this difficulty by considering strongly anisotropic 2D systems [71-74] formed by weakly coupled Rashba wires (see Fig. 1), where each of them can be treated as a one-dimensional Luttinger liquid by bosonization [75-86]. This will allow us to introduce the Floquet version not only of TIs but also of Weyl semimetals in driven 2D systems. Importantly, in this way we can also address fractional regimes and are able to obtain the Floquet version of fractional TIs and Weyl semimetals.
Topological dispersionless edge modes in driven systems. - We consider a 2D model formed by weakly tunnel-coupled Rashba wires, see Fig. 1. The spectrum of such one-dimensional wires consists of conduction and valence bands with Rashba SOIs, separated by a gap $\Delta_{g}$, and labeled by $\eta= \pm 1$, see Fig. 2. Semiconducting wires, atomic chains, as well as graphene and metal dichalcogenide nanoribbons can be used. However, for simplicity, we refer to all such single channel systems as wires in this work. The wires are aligned along the $x$ axes and lie in the $x y$ plane. The unit cells labeled by $n$ are composed of two wires with opposite SOIs labeled by $\lambda= \pm 1$. The kinetic part of the Hamiltonian (density) corresponding to the $\lambda$ wire in the $n$th unit cell is written as $H_{0 n \lambda}=$ $\sum_{\eta \sigma} \eta \Psi_{n \lambda \eta \sigma}^{\dagger}\left\{\left(\delta_{1 \eta} \Delta_{g}\right)-\left[\left(\hbar^{2} \partial_{x}^{2}\right) /\left(2 m_{0}\right)\right]+i \alpha \lambda \sigma \partial_{x}\right\} \Psi_{n \lambda \eta \sigma}$. Here, $m_{0}$ is the effective mass, and $\alpha$ is the strength of the SOI, with corresponding wave vector $k_{s o}=m_{0} \alpha / \hbar^{2}$ and energy $E_{s o}=\hbar^{2} k_{s o}^{2} / 2 m_{0}$. Without loss of generality, we choose the SOI vector to point in $z$ direction. Here, $\Psi_{n \lambda \eta \sigma}(x)$ is the annihilation operator acting on the particle of the $\eta$ band with spin $\sigma$, and located at position $x$ of the $\lambda$ wire in the $n$th unit cell. We assume the chemical potential $\mu$ to be tuned to the SOI induced crossing of the valence bands.

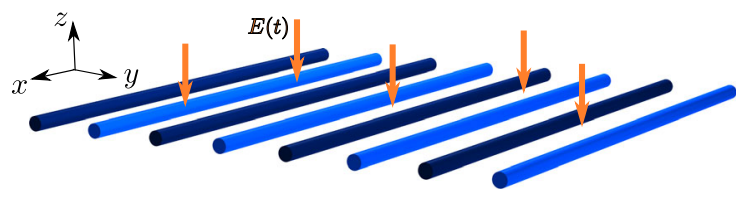

FIG. 1. A periodic array of weakly coupled Rashba wires (blue cylinders) aligned in the $x$ direction in the $x y$ plane. The sign of the SOI changes from positive (dark blue) to negative (light blue) inside the unit cell composed of two (four) wires in the first (second) model, see below. The driving field $E(t)$ with period $\omega$ applied along $z$ (orange arrows) results in a coupling of the conduction and valence bands (separated by a gap $\Delta_{g}$ ) at resonance when $\hbar \omega=\Delta_{g}$. 


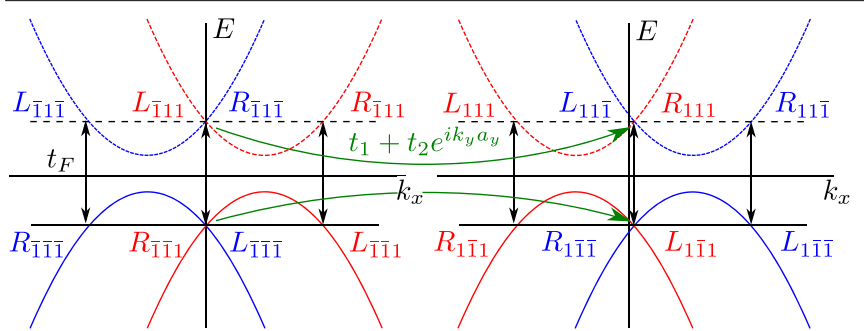

FIG. 2. The unit cell of the 2D system composed of two Rashba wires with opposite SOIs is shown in $k_{y}$-momentum space, where $R_{\lambda \eta \sigma} \equiv R_{k, \lambda \eta \sigma}$, etc. The gap $\Delta_{g}$ separates the valence $(\eta=\overline{1})$ and conduction $(\eta=1)$ bands. The index $\sigma=1(\sigma=\overline{1})$ refers to the spin up (spin down) band shown in red (blue). The chemical potential $\mu$ is tuned to $E_{s o}$ and the driving frequency is chosen as $\hbar \omega=\Delta_{g}$, resulting in resonant scattering (vertical black arrows) between bands with amplitude $t_{F}$, opening gaps. The interwire tunneling with amplitude $t_{1}+t_{2} e^{i k_{y} a_{y}}$ is shown by green arrows.

Next, we allow for an oscillating electric field, at frequency $\omega$, either due to an external electromagnetic radiation, or induced by periodically driving a voltage difference between a back and top gate enclosing the wire array. Driving at resonance across the band gap, that is with $\hbar \omega=\Delta_{g}$, opens a dynamical gap [87,88], essential for inducing nontrivial topology $[52,57,89]$. Using the Floquet representation [90-92], this gap arises as a splitting of degeneracies in the quasienergy spectrum of the Floquet operator. It corresponds to the lowest order of degenerate perturbation theory in the electric field amplitude, or, in another words, to a single photon emission or absorption process.

For simplicity, we work in the regime of strong SOIs and linearize the problem $[93,94]$ by representing operators in terms of spatially slowly varying left and right mover fields defined around the Fermi points $k_{F}=0, \pm 2 k_{s o}$ (see Fig. 2) as $\Psi_{n \lambda \eta \sigma}=R_{n \lambda \eta \sigma}(x) e^{i k_{s o}(\eta-\sigma \lambda) x}+L_{n \lambda \eta \sigma}(x) e^{-i k_{s o}(\eta+\sigma \lambda) x}$. The system is translation invariant in the $y$ direction, so we introduce the conserved momentum $k_{y}$ via $\Psi_{n}=$ $\sum_{k_{y}} e^{i n k_{y} a_{y}} \Psi_{k_{y}}$, where $a_{y}$ is the unit cell size. The kinetic term becomes $H_{0}=i \hbar v_{F} \sum_{k_{y} \lambda \eta \sigma}\left(L_{k_{y} \lambda \eta \eta \sigma}^{\dagger} \partial_{x} L_{k_{y} \lambda \eta \sigma}-\right.$ $\left.R_{k_{y} \lambda \eta \sigma}^{\dagger} \partial_{x} R_{k_{y} \lambda \eta \sigma \sigma}\right)$ with $v_{F}$ being the Fermi velocity. The interwire tunneling term, $H_{t}=\sum_{n \eta \sigma}\left(t_{1} \Psi_{n 1 \eta \sigma}^{\dagger} \Psi_{n \overline{1} \eta \sigma}+\right.$ $\left.t_{2} \Psi_{(n+1) 1 \eta \sigma}^{\dagger} \Psi_{n \overline{1} \eta \sigma}\right)+$ H.c., becomes in linearized form $H_{t}=\sum_{k_{y} \sigma} t_{y} e^{i \phi}\left(L_{k_{y} 1 \bar{\sigma} \sigma}^{\dagger} R_{k_{y} \overline{1} \bar{\sigma} \sigma}+R_{k_{y} 1 \sigma \sigma}^{\dagger} L_{k_{y} \overline{1} \sigma \sigma}\right)+$ H.c., where we keep only slowly oscillating terms. Here, we introduced $\left(t_{1}+t_{2} e^{i k_{y} a}\right) \equiv t_{y} e^{i \phi}$ with $t_{y}=\sqrt{t_{1}^{2}+t_{2}^{2}+2 t_{1} t_{2} \cos \left(k_{y} a_{y}\right)}$. The magnetic field is applied in-plane and perpendicular to the SOI vector, say, in the $x$ direction and is described by $H_{Z}=\Delta_{Z} \sum_{\eta \sigma \sigma^{\prime} n \lambda} \Psi_{n \lambda \eta \sigma}^{\dagger}\left(\sigma_{x}\right)_{\sigma \sigma^{\prime}} \Psi_{n \lambda \eta \sigma^{\prime}}$, yielding the linearized version

$$
H_{Z}=\Delta_{Z} \sum_{k_{y} \lambda \eta} R_{k_{y} \lambda \eta(\lambda \cdot \eta)}^{\dagger} L_{k_{y} \lambda \eta(\lambda \cdot \bar{\eta})}+\text { H.c. }
$$

The time-dependent driving term couples two bands and is given by $H_{F}=t_{F} \sum_{n \lambda \sigma} \Psi_{n \lambda 1 \sigma}^{\dagger} \Psi_{n \lambda \overline{1} \sigma}+$ H.c., or after linearization by

$$
H_{F}=t_{F} \sum_{n \lambda \eta \sigma} R_{n \lambda \eta \sigma}^{\dagger} L_{n \lambda \bar{\eta} \sigma}+\text { H.c., }
$$

where we neglected the fast-oscillating terms and the weak photon-assisted interwire intersubband scattering. The amplitude $t_{F}=e E d_{c v}$ is proportional to the interband dipole matrix element $d_{c v}$ and the $E$-field amplitude [95]. We consider the weak driving regime $t_{F} \ll \Delta_{q}$.

The bulk spectrum is given by $E_{1}^{2}=\left(\hbar v_{F} k_{x}\right)^{2}+t_{F}^{2}$ (fourfold degenerate) and $E_{2 \pm \pm}^{2}=\left(\hbar v_{F} k_{x}\right)^{2}+$ $\left(t_{F} \pm t_{y} \pm \Delta_{Z}\right)^{2}$. The bulk spectrum is gapless if $t_{y}=$ $\pm\left(t_{F} \pm \Delta_{Z}\right)$. For simplicity, we assume henceforth that all amplitudes are non-negative. There are two pairs of dispersionless zero-energy modes for $t_{F}<\left|t_{y}-\Delta_{Z}\right|$. If $\left|t_{y}-\Delta_{Z}\right|<t_{F}<\left|t_{y}+\Delta_{Z}\right|$, there is only one zero-energy mode. The system is trivial if $t_{F}>\left|t_{y}+\Delta_{Z}\right|$. The phase diagram recalculated in terms of the tunneling amplitudes $t_{1}$ and $t_{2}$ is shown in Fig. 3 and contains three topological phases. In two of them, the system is fully gapped and hosts $\nu=1(\nu=2)$ dispersionless modes, green (yellow) area in Fig. 3, where $\nu$ refers to Chern numbers [105]. Interestingly, there emerges also a topological phase similar to Weyl semimetals (blue area in Fig. 3) [13-17], see Fig. 4. Indeed, the spectrum is gapless due to two (possibly four) Dirac cones located at $\pm k_{ \pm}$, defined by the condition for closing the bulk gap, $\cos \left(k_{ \pm} a\right)=\left[\left(t_{F} \pm \Delta_{Z}\right)^{2}-\right.$ $\left.t_{1}^{2}-t_{2}^{2}\right] / 2 t_{1} t_{2}$. Following Refs. [106,107], we confirmed that the Dirac cones have opposite chiralities as expected for the Weyl phase, see the Supplemental Material [95]. In addition, there are dispersionless zero-energy edge modes (Fermi arcs) that connect the Dirac cones, see e.g., Fig. 4(b). These modes represent 1D edge channels, in contrast to 2D surface states typical for 3D systems [86]. The particle-hole symmetry guarantees edge states to be at zero energy. For details on the wave functions we refer to

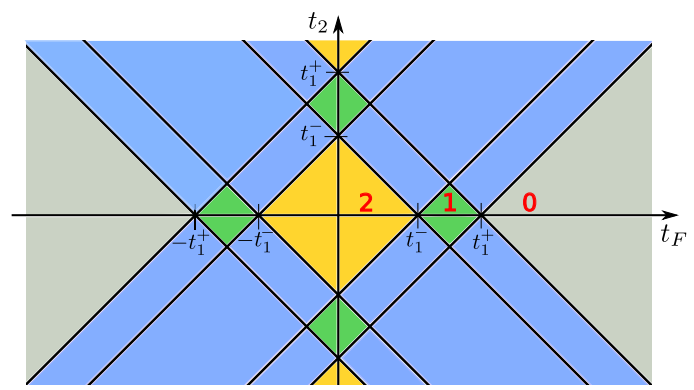

FIG. 3. The phase diagram of the model shown in Fig. 2 as a function of Floquet coupling $t_{F}$ and tunneling amplitude $t_{2}$. For fixed values of the tunneling amplitude $t_{1}$ and the Zeeman energy $\Delta_{Z}$ we determine $t_{1}^{ \pm}=t_{1} \pm \Delta_{Z}$ (here, we assume that $\Delta_{Z}<t_{1}$ such that $t_{1}^{-}>0$ ). The system in the topological phase is gapped in the bulk and hosts either one zero-energy edge mode (green area, $\nu=1$ ) or two zero-energy edge modes (yellow area, $\nu=2$ ). In contrast, in the trivial phase, the system does not support edge modes in the gap (gray area, $\nu=0$ ). In the Weyl phase (blue area), edge modes connect two gapless bulk cones, see Fig. 4(b). 

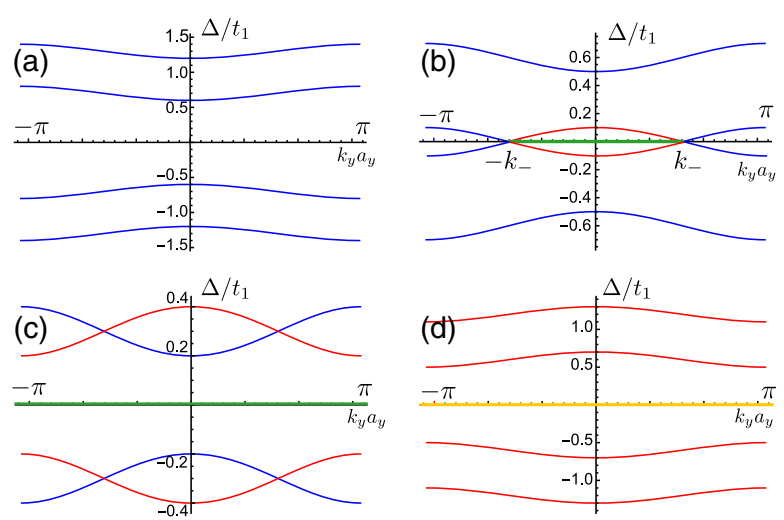

FIG. 4. The dependence of the minimum bulk gap $\Delta$ of $E_{2 \pm \pm}$ occurring at $k_{x}=0$ on the transverse momentum $k_{y}$. The parameters are chosen as $t_{2} / t_{1}=0.1, \Delta_{Z} / t_{1}=0.3$. (a) The system is in the trivial phase for $t_{F} / t_{1}=2$. (b) As $t_{F}$ decreases, $t_{F} / t_{1}=1.4$, the bulk gap closes at $k_{y}= \pm k_{-}$, and a single zeroenergy mode at each edge (green line) connects two Dirac cones (bulk spectrum). (c) For $t_{F} / t_{1}=1$, the bulk gap reopens and leaves one of the bands (red lines) in the topological phase with a single zero-energy mode at each edge. (d) At small $t_{F}$, $t_{F} / t_{1}=0.1$, both bands are in the topological phase resulting in two zero-energy modes at each edge (yellow line). See Fig. 3 for the phase description.

the Supplemental Material [95], where we also discuss the extension to fractional Weyl semimetals. However, flat bands are fragile upon adding electron-electron interactions; thus, we need to exclude the formation of, e.g., a charge-density wave such that one can assume $k_{y}$ to stay a good quantum number.

Floquet TI.-The model considered above hosts only zero-energy edge modes. In our second model we propose a setup that will host helical edge modes. To achieve this, we need to break the symmetry between hoppings along the edge in positive and negative directions. This can be implemented with a unit cell consisting of four wires, labeled by $\lambda, \tau= \pm 1$, see Fig. 5 . The sign of the Rashba SOI $\alpha_{\lambda} \equiv \lambda \alpha$ alternates between wires as well as the band gap $\Delta_{g \tau \lambda}=\Delta_{g}+\tau(1-\lambda) \delta / 2$. The chemical potential is tuned to the SOI energy inside each wire and the driving frequency matches the resonance between the Fermi level and the conduction band with the same momentum, $\hbar \omega_{\tau \lambda}=\Delta_{g \tau \lambda}+2 E_{s o}$, see Fig. 5. We note that the bias between the wires due to different chemical potentials results in a small leakage current, which we estimate in Ref. [95]. Because of translation invariance along the wire, the interwire tunneling is assumed to be momentum and spin conserving. For simplicity, we assume that all interwire tunneling amplitudes are equal. In the limit $t_{1} \gg t_{F}$, we perform the perturbation in two steps. First, as before, we linearize the Hamiltonian close to the Fermi level. The operators $\Psi_{n \tau \lambda \eta \sigma}$ are represented in terms of left $L_{n \tau \lambda \eta \sigma}$ and right $R_{n \tau \lambda \eta \sigma}$ movers. In the tunneling term we keep only resonant slow-varying terms,

$$
\begin{aligned}
H_{1}= & t_{1} \sum_{n \tau \lambda} R_{n \tau \lambda \overline{1} \lambda}^{\dagger} L_{n \tau \bar{\tau} \overline{1} \lambda}+t_{1} \sum_{n \tau} R_{n \tau \bar{\tau} 1 \tau}^{\dagger} L_{n \bar{\tau} \tau 1 \tau} \\
& +t_{1} \sum_{n \tau}\left(R_{n \overline{1} \overline{1} 11}^{\dagger} L_{(n+1) 1111}+L_{n \overline{1} \overline{1} 1 \overline{1}}^{\dagger} R_{(n+1) 111 \overline{1}}\right) \\
& + \text { H.c. }
\end{aligned}
$$

All modes appearing in $H_{1}$ are gapped out with a gap of size $t_{1}$. The remaining modes are gapped out by the periodic driving. We note that here we can use the Floquet technique for each wire independently. The corresponding Hamiltonian density is given by

$$
H_{F}=t_{F} \sum_{n \tau \lambda \eta} R_{n \tau \lambda \eta \eta(\eta \lambda)}^{\dagger} L_{n \tau \lambda \bar{\eta}(\eta \lambda)}+\text { H.c. }
$$

Again, the bulk modes in $H_{F}$ are gapped out. However, there are two modes, one at each edge wire, that are neither coupled by $H_{1}$ nor by $H_{F}$ to the rest. For example, if the system is composed out of an integer number of unit cells $N$, there are two gapless modes protected by time-reversal symmetry with opposite spins and velocities at each of the two edge wires. The most right (left) wire hosts helical edge modes $L_{(n=1) 1111}$ and $R_{(n=1) 111 \overline{1}} \quad\left(L_{(n=N) \overline{1} \overline{1} 1 \overline{1}} \quad\right.$ and

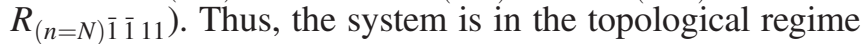
and represents the Floquet version of a two-dimensional TI.

Fractional Floquet TI.-The same model as in the preceding section can also be brought into the fractional regime. To get, for example, the effective filling factor $\nu=1 / 3$, we tune the chemical potential down to $E_{s o} / 9$ $[78,83]$. However, in this case, the interwire tunneling is possible only if backscattering events due to electronelectron interactions are involved. We note that Floquet representation remains unchanged also in the presence of interactions since they commute with the driving field operator and the driving frequency $\omega$ sets the fastest time scale in the system. The interactions can be treated nonperturbatively by standard bosonization: $R_{n \tau \lambda \eta \sigma}=e^{i \phi_{n 1 \tau \lambda \eta \sigma}}$ and $L_{n \tau \lambda \eta \eta \sigma}=e^{i \phi_{n \bar{\tau} \tau \lambda \eta \sigma}}$, and by introducing new bosonic fields $\tilde{\phi}_{n r \tau \lambda \eta \sigma \sigma}=\left(2 \phi_{n r \tau \lambda \eta \sigma}-\phi_{n \bar{r} \tau} \lambda \eta \sigma \sigma\right) / 3$. Next, we use a two-step perturbation procedure and assume that the tunneling term describes the dominant process. This process involves the back-scattering of two electrons and is the lowest order process in $t_{1}$ that satisfies both spin and momentum conservation, where $g_{B}$ is the electron-electron back-scattering amplitude [45,46,48],

$$
\begin{aligned}
H_{1}^{e e}= & g_{1} \sum_{n \tau \lambda} \cos \left[3\left(\tilde{\phi}_{n 1 \tau \lambda \overline{1} \lambda}-\tilde{\phi}_{n \overline{1} \bar{\tau} \bar{\lambda} \lambda}\right)\right] \\
& +g_{1} \sum_{n \tau} \cos \left[3\left(\tilde{\phi}_{n 1 \overline{1} \overline{1} \tau}-\tilde{\phi}_{n \overline{1} \bar{\tau} \tau 1 \tau}\right)\right] \\
& +g_{1} \sum_{n}\left(\cos \left[3\left(\tilde{\phi}_{n 1 \overline{1} \overline{1} 11}-\tilde{\phi}_{(n+1) \overline{1} 1111}\right)\right]\right. \\
& \left.+\cos \left[3\left(\tilde{\phi}_{(n+1) 1111 \overline{1}}-\tilde{\phi}_{n \overline{1} \overline{1} \overline{1} 1 \overline{1}}\right)\right]\right) .
\end{aligned}
$$


(a)

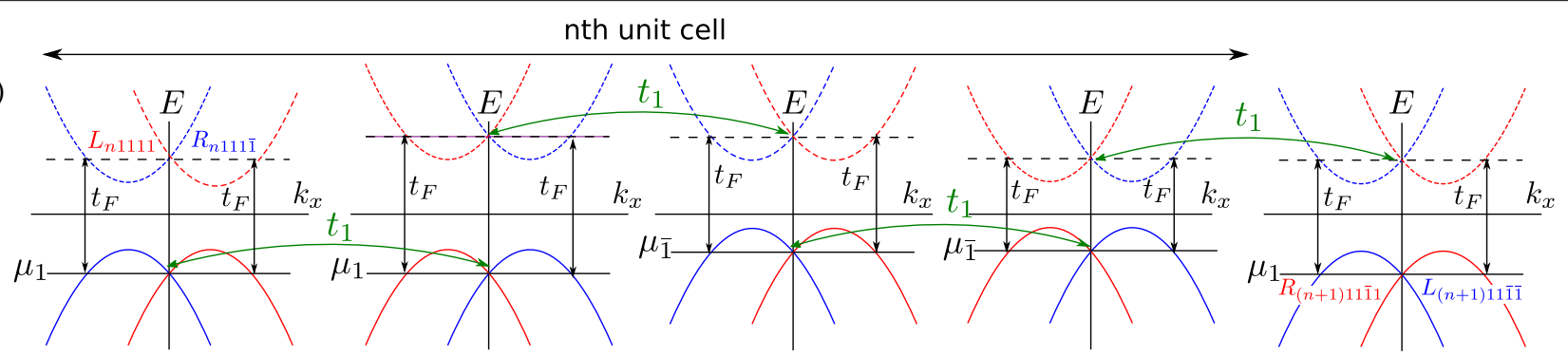

$$
\Delta_{g} ; \tau=1 ; \lambda=1
$$

$\Delta_{g}^{+} ; \tau=1 ; \lambda=\overline{1}$

$\Delta_{g} ; \tau=\overline{1} ; \lambda=1$

$$
\Delta_{g}^{-} ; \tau=\overline{1} ; \lambda=\overline{1}
$$

$\Delta_{g} ; \tau=1 ; \lambda=1$

(b)

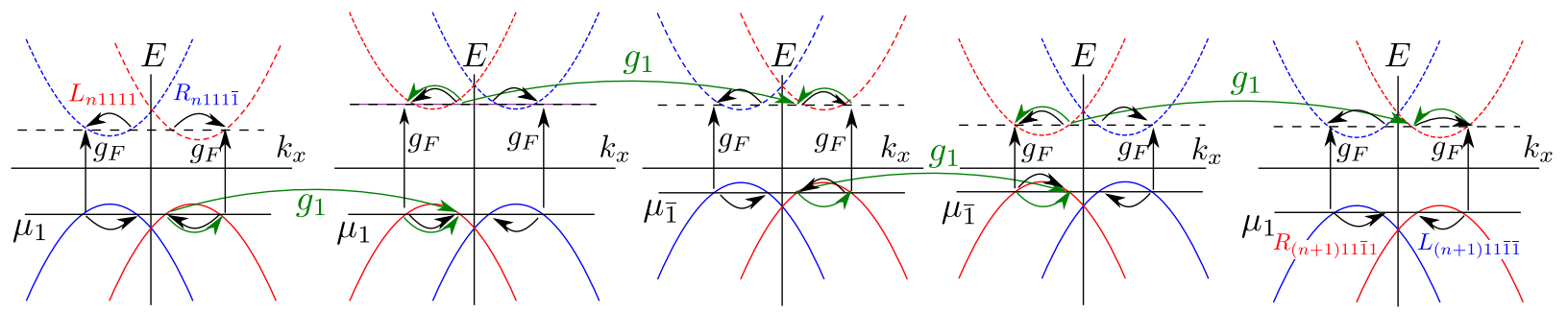

FIG. 5. The unit cell of a Floquet TI, consisting of four wires labeled by $\tau, \lambda= \pm 1$. The sign of the SOI alternates between wires while the band gap alternates between $\Delta_{g}$ and $\Delta_{g \pm}=\Delta_{g} \pm \delta$. (a) In the integer regime, $\mu_{\tau}$ is tuned to $E_{s o}$ and the driving frequencies are chosen to match the band gaps. The interwire hopping of amplitude $t_{1}$ opens gaps at $k_{x}=0$ if resonant. The remaining gaps of size $t_{F}$ are opened by the time-dependent driving. At each edge of the array, there is a pair of helical modes left gapless. For example, if the system is terminated as shown in this figure, the modes $L_{n 1111}$ and $R_{n 111 \overline{1}}$ at the left edge stay gapless. (b) In the fractional regime, $\mu_{\tau}$ is shifted to $E_{s o} / 9$ and the driving frequency is readjusted accordingly. The leading term in the tunneling between wires $H_{1}^{e e}$ (green arrows) of amplitude $g_{1}$ involves two back-scattering events and opens a partial gap due to strong interactions. The driving term $H_{F}^{e e}$ of amplitude $g_{F}$ (black arrows) commutes with $H_{1}^{e e}$ and both can order simultaneously.

Again, the driving frequency matches the energy difference between the bands, see Fig. 5. For weak driving it is sufficient to include the electron-electron interactions inside each band. The term that commutes with $H_{1}^{e e}$ and satisfies the conservation laws such that it could be ordered simultaneously in the renormalization group sense $[45,46,48]$ leads to a gap and is given by

$$
H_{F}^{e e}=g_{F} \sum_{n \tau \lambda \eta} \cos \left[3\left(\tilde{\phi}_{n 1 \tau \lambda \eta(\eta \lambda)}-\tilde{\phi}_{n \overline{1} \tau \lambda \bar{\eta}(\eta \lambda)}\right)\right]
$$

Here, the amplitude $g_{1} \propto t_{1} g_{B}^{2}\left(g_{F} \propto t_{F} g_{B}^{2}\right)$ depends on the amplitude $g_{B}$ of back-scattering processes. Similarly to the integer case, two helical fields at each edge (for example, $\tilde{\phi}_{(n=1) \overline{1} 1111}$ and $\tilde{\phi}_{(n=1) 1111 \overline{1}}$ in the first unit cell) do not enter into the coupling Hamiltonians and stay gapless. In addition, elementary excitations in these modes have fractional charge $e / 3$. All this shows that we have generated a Floquet version of a fractional TI. The same procedure can be straightforwardly generalized to other odd integer values $m$, giving rise to fractional Floquet TIs with fractional excitations $e / m$.

Conclusions. - We have studied arrays of weakly coupled Rashba wires brought into the topological regime by periodic driving. Our first model hosts dispersionless zero-energy modes and may be identified with Weyl semimetals. The second model is in the TI regime and hosts helical edge modes, where the elementary excitations have fractional charge $e / m$ with $m$ being an odd integer. The proposed effects can be measured in transport experiments (by observing their propagation [66-68] or localization [69] at the edge) or via the density of states $[50-52,55]$. It is worth noting that the same models can be obtained without driving but instead by doubling the wires [108]. Finally, nonequilibrium heating and relaxation mechanisms producing mobile bulk quasiparticles [109-111], issues generally pertinent to Floquet systems [112], are important here as well. It has been argued that these difficulties can be overcome by, e.g., adiabatic buildup of states [52,113] or engineered baths [114].

We acknowledge support from the Swiss NSF, NCCR QSIT (National Center of Competence in Research "Quantum Science and Technology"), and JSPS KAKENHI Grant No. 16H02204.

[1] M. Z. Hasan and C. L. Kane, Rev. Mod. Phys. 82, 3045 (2010).

[2] X.-L. Qi and S.-C. Zhang, Rev. Mod. Phys. 83, 1057 (2011).

[3] G. Tkachov and E. M. Hankiewicz, Phys. Status Solidi B 250, 215 (2013).

[4] B. A. Volkov and O. A. Pankratov, Pis'ma Zh. Eksp. Teor. Fiz. 42, 145 (1985) [JETP Lett. 42, 178 (1985)].

[5] O. A. Pankratov, S. V. Pakhomov, and B. A. Volkov, Solid State Commun. 61, 93 (1987). 
[6] B. A. Bernevig, T. L. Hughes, and S.-C. Zhang, Science 314, 1757 (2006).

[7] M. König, S. Wiedmann, C. Brune, A. Roth, H. Buhmann, L. W. Molenkamp, X.-L. Qi, and S.-C. Zhang, Science 318, 766 (2007).

[8] A. Roth, C. Brune, H. Buhmann, L. W. Molenkamp, J. Maciejko, X.-L. Qi, and S.-C. Zhang, Science 325, 294 (2009).

[9] Y. Tanaka, T. Yokoyama, and N. Nagaosa, Phys. Rev. Lett. 103, 107002 (2009).

[10] K. C. Nowack, E. M. Spanton, M. Baenninger, M. König, J. R. Kirtley, B. Kalisky, C. Ames, P. Leubner, C. Brune, H. Buhmann, L. W. Molenkamp, D. Goldhaber-Gordon, and K. A. Moler, Nat. Mater. 12, 787 (2013).

[11] S. Hart, H. Ren, T. Wagner, P. Leubner, M. Muhlbauer, C. Brune, H. Buhmann, L. W. Molenkamp, and A. Yacoby, Nat. Phys. 10, 638 (2014).

[12] V. S. Pribiag, A. J. A. Beukman, F. Qu, M. C. Cassidy, C. Charpentier, W. Wegscheider, and L. P. Kouwenhoven, Nat. Nanotechnol. 10, 593 (2015).

[13] X. Wan, A. M. Turner, A. Vishwanath, and S. Y. Savrasov, Phys. Rev. B 83, 205101 (2011).

[14] K.-Y. Yang, Y.-M. Lu, and Y. Ran, Phys. Rev. B 84, 075129 (2011).

[15] A. A. Burkov and L. Balents, Phys. Rev. Lett. 107, 127205 (2011).

[16] G. Xu, H. Weng, Z. Wang, X. Dai, and Z. Fang, Phys. Rev. Lett. 107, 186806 (2011).

[17] A. A. Zyuzin and A. A. Burkov, Phys. Rev. B 86, 115133 (2012).

[18] M. Sato and S. Fujimoto, Phys. Rev. B 79, 094504 (2009).

[19] R. M. Lutchyn, J. D. Sau, and S. Das Sarma, Phys. Rev. Lett. 105, 077001 (2010).

[20] Y. Oreg, G. Refael, and F. von Oppen, Phys. Rev. Lett. 105, 177002 (2010).

[21] J. Alicea, Phys. Rev. B 81, 125318 (2010).

[22] A. C. Potter and P. A. Lee, Phys. Rev. B 83, 094525 (2011).

[23] J. Klinovaja, S. Gangadharaiah, and D. Loss, Phys. Rev. Lett. 108, 196804 (2012).

[24] D. Chevallier, D. Sticlet, P. Simon, and C. Bena, Phys. Rev. B 85, 235307 (2012).

[25] D. Sticlet, C. Bena, and P. Simon, Phys. Rev. Lett. 108, 096802 (2012).

[26] J. Klinovaja, P. Stano, and D. Loss, Phys. Rev. Lett. 109, 236801 (2012).

[27] S. Nadj-Perge, I. K. Drozdov, B. A. Bernevig, and A. Yazdani, Phys. Rev. B 88, 020407(R) (2013).

[28] J. Klinovaja, P. Stano, A. Yazdani, and D. Loss, Phys. Rev. Lett. 111, 186805 (2013).

[29] B. Braunecker and P. Simon, Phys. Rev. Lett. 111, 147202 (2013).

[30] M. M. Vazifeh and M. Franz, Phys. Rev. Lett. 111, 206802 (2013).

[31] V. Mourik, K. Zuo, S. M. Frolov, S. R. Plissard, E. P. A. M. Bakkers, and L. P. Kouwenhoven, Science 336, 1003 (2012).

[32] M. T. Deng, C. L. Yu, G. Y. Huang, M. Larsson, P. Caroff, and H. Q. Xu, Nano Lett. 12, 6414 (2012).
[33] A. Das, Y. Ronen, Y. Most, Y. Oreg, M. Heiblum, and H. Shtrikman, Nat. Phys. 8, 887 (2012).

[34] L. P. Rokhinson, X. Liu, and J. K. Furdyna, Nat. Phys. 8, 795 (2012).

[35] J. R. Williams, A. J. Bestwick, P. Gallagher, S. S. Hong, Y. Cui, A. S. Bleich, J. G. Analytis, I. R. Fisher, and D. Goldhaber-Gordon, Phys. Rev. Lett. 109, 056803 (2012).

[36] H. O. H. Churchill, V. Fatemi, K. Grove-Rasmussen, M. T. Deng, P. Caroff, H. Q. Xu, and C. M. Marcus, Phys. Rev. B 87, 241401(R) (2013).

[37] S. Nadj-Perge, I. K. Drozdov, J. Li, H. Chen, S. Jeon, J. Seo, A. H. MacDonald, B. A. Bernevig, and A. Yazdani, Science 346, 602 (2014).

[38] R. Pawlak, M. Kisiel, J. Klinovaja, T. Meier, S. Kawai, T. Glatzel, D. Loss, and E. Meyer, arXiv:1505.06078.

[39] M. Barkeshli, C, M. Jian, and X.-L. Qi, Phys. Rev. B 87, 045130 (2013).

[40] N. H. Lindner, E. Berg, G. Refael, and A. Stern, Phys. Rev. X 2, 041002 (2012).

[41] D. Clarke, J. Alicea, and K. Shtengel, Nat. Commun. 4, 1348 (2013).

[42] M. Cheng, Phys. Rev. B 86, 195126 (2012).

[43] R. S. K. Mong, D. J. Clarke, J. Alicea, N. H. Lindner, P. Fendley, C. Nayak, Y. Oreg, A. Stern, E. Berg, K. Shtengel, and M. P. A. Fisher, Phys. Rev. X 4, 011036 (2014).

[44] A. Vaezi, Phys. Rev. X 4, 031009 (2014).

[45] J. Klinovaja and D. Loss, Phys. Rev. Lett. 112, 246403 (2014).

[46] J. Klinovaja and D. Loss, Phys. Rev. B 90, 045118 (2014).

[47] J. Klinovaja, A. Yacoby, and D. Loss, Phys. Rev. B 90, 155447 (2014).

[48] Y. Oreg, E. Sela, and A. Stern, Phys. Rev. B 89, 115402 (2014).

[49] C. P. Orth, R. P. Tiwari, T. Meng, and T. L. Schmidt, Phys. Rev. B 91, 081406(R) (2015).

[50] T. Oka and H. Aoki, Phys. Rev. B 79, 081406 (2009).

[51] T. Kitagawa, E. Berg, M. Rudner, and E. Demler, Phys. Rev. B 82, 235114 (2010).

[52] N. H. Lindner, G. Refael, and V. Galitski, Nat. Phys. 7, 490 (2011).

[53] L. Jiang, T. Kitagawa, J. Alicea, A. R. Akhmerov, D. Pekker, G. Refael, J. I. Cirac, E. Demler, M. D. Lukin, and P. Zoller, Phys. Rev. Lett. 106, 220402 (2011).

[54] M. Trif and Y. Tserkovnyak, Phys. Rev. Lett. 109, 257002 (2012).

[55] N. H. Lindner, D. L. Bergman, G. Refael, and V. Galitski, Phys. Rev. B 87, 235131 (2013).

[56] J. I. Inoue and A. Tanaka, Phys. Rev. Lett. 105, 017401 (2010).

[57] M. S. Rudner, N. H. Lindner, E. Berg, and M. Levin, Phys. Rev. X 3, 031005 (2013).

[58] D. E. Liu, A. Levchenko, and H. U. Baranger, Phys. Rev. Lett. 111, 047002 (2013).

[59] A. A. Reynoso and D. Frustaglia, Phys. Rev. B 87, 115420 (2013).

[60] M. Thakurathi, K. Sengupta, and D. Sen, Phys. Rev. B 89, 235434 (2014). 
[61] A. G. Grushin, A. Gomez-Len, and T. Neupert, Phys. Rev. Lett. 112, 156801 (2014).

[62] M. Thakurathi, A. A. Patel, D. Sen, and A. Dutta, Phys. Rev. B 88, 155133 (2013).

[63] P. Delplace, A. Gomez-Leon, and G. Platero, Phys. Rev. B 88, 245422 (2013).

[64] Y. T. Katan and D. Podolsky, Phys. Rev. Lett. 110, 016802 (2013).

[65] A. Kundu and B. Seradjeh, Phys. Rev. Lett. 111, 136402 (2013).

[66] K. Fang, Z. Yu, and S. Fan, Nat. Photonics 6, 782 (2012).

[67] P. M. Perez-Piskunow, G. Usaj, C. A. Balseiro, and L. E. F. Foa Torres, Phys. Rev. B 89, 121401(R) (2014).

[68] L. E. F. Foa Torres, P. M. Perez-Piskunow, C. A. Balseiro, and G. Usaj, Phys. Rev. Lett. 113, 266801 (2014).

[69] T. Kitagawa, M. A. Broome, A. Fedrizzi, M. S. Rudner, E. Berg, I. Kassal, A. Aspuru-Guzik, E. Demler, and A. G. White, Nat. Commun. 3, 882 (2012).

[70] A. Agarwala, U. Bhattacharya, A. Dutta, and D. Sen, arXiv:1602.08195.

[71] A. G. Lebed, JETP Lett. 43, 174 (1986).

[72] V. M. Yakovenko, Phys. Rev. B 43, 11353 (1991).

[73] C. L. Kane, R. Mukhopadhyay, and T. C. Lubensky, Phys. Rev. Lett. 88, 036401 (2002).

[74] J. Klinovaja and D. Loss, Phys. Rev. Lett. 111, 196401 (2013).

[75] J. C. Y. Teo and C. L. Kane, Phys. Rev. B 89, 085101 (2014).

[76] J. Klinovaja and D. Loss, Eur. Phys. J. B 87, 171 (2014).

[77] T. Meng, P. Stano, J. Klinovaja, and D. Loss, Eur. Phys. J. B 87, 203 (2014).

[78] J. Klinovaja and Y. Tserkovnyak, Phys. Rev. B 90, 115426 (2014).

[79] T. Neupert, C. Chamon, C. Mudry, and R. Thomale, Phys. Rev. B 90, 205101 (2014).

[80] I. Seroussi, E. Berg, and Y. OregPhys. Rev. B 89, 104523 (2014).

[81] E. Sagi and Y. Oreg, Phys. Rev. B 90, 201102 (2014).

[82] T. Meng and E. Sela, Phys. Rev. B 90, 235425 (2014).

[83] J. Klinovaja, Y. Tserkovnyak, and D. Loss, Phys. Rev. B 91, 085426 (2015).

[84] G. Gorohovsky, R. G. Pereira, and E. Sela, Phys. Rev. B 91, 245139 (2015).

[85] R. A. Santos, C.-W. Huang, Y. Gefen, and D. B. Gutman, Phys. Rev. B 91, 205141 (2015).

[86] T. Meng, Phys. Rev. B 92, 115152 (2015).

[87] Y. H. Wang, H. Steinberg, P. Jarillo-Herrero, and N. Gedik, Science 342, 453 (2013).

[88] F. H. M. Faisal and J. Z. Kamiński, Phys. Rev. A 56, 748 (1997).

[89] T. Kitagawa, T. Oka, A. Brataas, L. Fu, and E. Demler, Phys. Rev. B 84, 235108 (2011).

[90] J. H. Shirley, Phys. Rev. 138, B979 (1965).
[91] P. Stano, J. Klinovaja, F. R. Braakman, L. M. K. Vandersypen, and D. Loss, Phys. Rev. B 92, 075302 (2015).

[92] H. Sambe, Phys. Rev. A 7, 2203 (1973).

[93] B. Braunecker, G. I. Japaridze, J. Klinovaja, and D. Loss, Phys. Rev. B 82, 045127 (2010).

[94] J. Klinovaja and D. Loss, Phys. Rev. B 86, 085408 (2012).

[95] See Supplemental Material at http://link.aps.org/ supplemental/10.1103/PhysRevLett.116.176401 for details on edge mode wave functions, Floquet formalism, and Weyl semimetals in the fractional regime, which includes Refs. [99-107].

[96] T. Karzig, Ch.-E. Bardyn, N. H. Lindner, and G. Refael, Phys. Rev. X 5, 031001 (2015).

[97] Z. Gu, H. A. Fertig, D. P. Arovas, and A. Auerbach, Phys. Rev. Lett. 107, 216601 (2011).

[98] T. Meng, J. Klinovaja, and D. Loss, Phys. Rev. B 89, 205133 (2014).

[99] L. V. Butov, C. W. Lai, A. L. Ivanov, A. C. Gossard, and D. S. Chemla, Nature (London) 417, 47 (2002).

[100] C. W. Lai, J. Zoch, A. C. Gossard, and D. S. Chemla, Science 303, 503 (2004).

[101] A. V. Nalitov, M. Vladimirova, A. V. Kavokin, L. V. Butov, and N. A. GippiusPhys. Rev. B 89, 155309 (2014).

[102] P. Andreakou, S. Cronenberger, D. Scalbert, A. Nalitov, N. A. Gippius, A. V. Kavokin, M. Nawrocki, J. R. Leonard, L. V. Butov, K. L. Campman, A. C. Gossard, and M. Vladimirova, Phys. Rev. B 91, 125437 (2015).

[103] J. D. Jackson, Classical Electrodynamics (Wiley, New York, 1975), p. 80.

[104] T. Giamarchi, Quantum Physics in One Dimension (Oxford University Press, Oxford, 2004).

[105] S. Ruy, A. Schnyder, A. Furusaki, and A. W. W. Ludwig, New J. Phys. 12, 065010 (2010).

[106] J.-M. Hou, Phys. Rev. Lett. 111, 130403 (2013).

[107] K. Sun, W. V. Liu, A. Hemmerich, and S. Das Sarma, Nat. Phys. 8, 67 (2012).

[108] The former valence band is represented by one wire and the conduction band by a subsequent one, and so on, with the $\mu$ 's tuned to resonance. The interwire tunneling results in the same Hamiltonian as via Floquet driving. However, simple tunneling is more susceptible to disorder, breaking the symmetry between bands. Thus, the Floquet driving seems experimentally more realistic.

[109] G. M. Eliashberg, JETP Lett. 11, 114 (1970).

[110] L. I. Glazman, Sov. Phys. JETP 53, 178 (1981).

[111] H. Dehghani, T. Oka, and A. Mitra, Phys. Rev. B 90, 195429 (2014).

[112] T. Iadecola, T. Neupert, and C. Chamon, Phys. Rev. B 91, 235133 (2015).

[113] L. E. F. Foa Torres, P. M. Perez-Piskunow, C. A. Balseiro, and G. Usaj, Phys. Rev. Lett. 113, 266801 (2014).

[114] K. I. Seetharam, C.-E. Bardyn, N. H. Lindner, M. S. Rudner, and G. Refael, Phys. Rev. X 5, 041050 (2015). 